\title{
Dynamic characteristics of dual-rotor system with coupling faults of misalignment and rub-impact
}

\author{
Zhiwei Huang* \\ China Ship Developmentand Design Center, Wuhan 430064, China
}

\begin{abstract}
According to fault problems of the rotor system local rubbing caused by mass eccentricity, a dynamic model for the dual-rotor system with coupling faults of misalignment and rub-impact was established. The dynamic behaviours of this system were investigated by using numerical integral method, as parallel and angular misalignment varied. Various nonlinear phenomena compressing periodic, threeperiodic and quasi-periodic motions are observed. The results reveal that the process of the rotor rub-impact is extremely complex and has some frequencies with large amplitude, especially at the $1 / 3 \mathrm{X}$ component. Meanwhile, quasi-periodic regions exhibit different configurations of attractors and the phenomenon of beat vibration.
\end{abstract}

\section{Introduction}

Rotor misalignment is one of the most common difficulties in the operation of rotating machinery, yet it remains incompletely understood. Despite the rapid increase in understanding of rotor dynamics, no satisfactory analysis explains the range of observed phenomena. At a time when the rotor dynamics may be regarded as a mature technology, misalignment remains as an outstanding area, where basic understanding is somewhat lacking [1]. Part of the difficulty may arise because the characteristics of misalignment cover a range of situations and the resulting behaviour arises from a combination of physical process. Under the misaligned condition, a series of negative dynamic effects such as wear of bearing, bending deformation of shafts, rotor rub-impact and so on [2-4], would be induced during the motion process of equipment. The rotor unbalance contributed to the misalignment of the journal bearing is always existent [5]. To achieve maximum efficiency in rotating machinery, it is essential to minimize the clearance between the rotor and stator. As a result, the rub phenomenon is also one of the main problems [6].

There are many types of industrial couplings and some of which are of rigid, gear and flexible type. The couplings' function is to transmit torque from the driver to the driven machine, There are two types of coupling misalignments: parallel and angular. A combination of parallel and angular misalignment is common in the industry [7]. In the past several years, efforts have been dedicated on studying and understanding the nonlinear phenomena in rotating machinery with the rotor misalignment or rub-impact. According to the characteristics of the coupling and the present situation in researching on the mechanis m of misalignment both at home and abroad, the misalignment was classified in detail by Xia [8]. Hu [9] studied a rig for testing lateral misalignment effects in a flexible rotor supported on three or more hydrodynamic journal bearings. The kinetic mechanism of the rotor system connected by the gear coupling and its faults physical properties were researched by $\mathrm{Li}$ [2] and Han [10] form the view point of kinematics when they are of misalignment. The dynamic and characteristics of vibration malfunction of various kinds of shaft misalignment were discussed by Wang [11]. Lees [1] reported some early studies on rotor misalignment and clearly the work had been concentrated on a single form of misalignment. Recent analytical and numerical simulations by Al-Hussain [12] and Bouaziz [13] show the influence of angular misalignment on the dynamic behaviour of a rotor system. From experimental investigations on misalignment rotor systems, frequency spectrum with series of harmonics of running speed was reported by Saavedra and Ramirez [14]. It was also reported [15] that steady-state vibration response at integer fraction of the first bending natural frequency is investigated. Analys is on complicated characteristics of the rotor system with rub-impact has been studied comprehensively [16-18]. Many practical analytical methods were also developed to analyze and interpret the results. Despite a significant number of theoretical and experiment investigations were conducted so far, most of the previous research was focused on gear coupling and horizontal unit with the parallel misalignment. Moreover, little attention was given to the rotor rub-impact due to rigid coupling misalignment that contains both parallel misalignment and angular misalignment. Dynamic behaviours of the rotor system with coupling faults are more complication than that with only one fault. Hence, intensive on the mechanis $\mathrm{m}$ and phenomena of nonlinear vibration

\footnotetext{
Corresponding author: hzwhust $@$ aliyun.com
} 
coupled faults with parallel misalignment and rub-impact in the rotor system has become one of the key issues for accurate fault diagnosis, dynamic design and control.

To make a profound understanding of the dynamic characteristics and malfunctions of rotating machinery with coupling faults of misalign ment and rub-impact, the paper presents a model of the dual-rotor system with coupling faults and analyzes its dynamic characteristics by using modern nonlinear dynamics and rotor dynamics theories. For different vib ration types of the rotor system, it shows different phenomena and dynamic behaviours, such as periodic, period-three and quasi-periodic. It is indicated that this study can contribute to a further understanding of the non-linear dynamics of such a rotor system with coupling faults of misalignment and rubimpact.

\section{Dynamic model of the dual-rotor system}

Rigid coupling by which the rotor 1 and rotor 2 are connected together cannot compensate the relative displacement of the two shafts and force the rotor 1 to follow the rotor 2. The motion process of the rotor misalignment and rub-impact is very complicated, involving a lot of factors. In order to make the main problem outstanding, the mechanical model can be simplified as shown in Fig.1. $O_{1}$ and $O_{2}$ are the center of the rotor 1 and rotor 2 , and $m_{1}$ and $m_{2}$ with eccentricity $e_{1}$ and $e_{2}$ are the masses of the rotor 1 and rotor 2, respectively. Their radial displacements are assumed as $\left(x_{1}, y_{1}\right)$ and $\left(x_{2}, y_{2}\right)$, respectively.

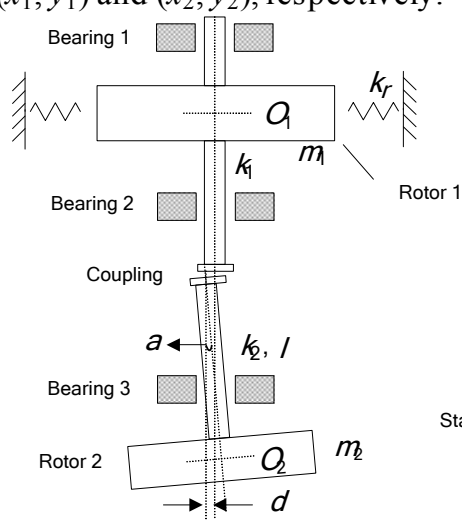

(a)

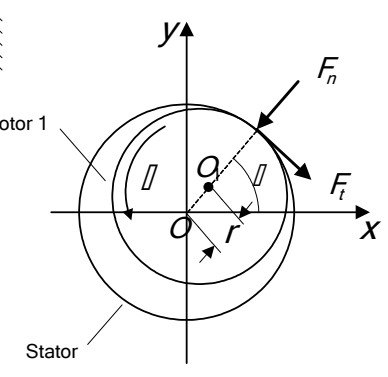

(b)
Fig. 1. Mechanical model of the dual-rotor system and schematic diagram of rotor rub-impact

To analyze above the dual-rotor system, the following assumptions are made:

(a) the masses of the two rotors are lumped at mid-point;

(b) the rotors, shafts and bearings are rigid and symmetric;

(c) axial and torsional vibration are negligible;

(d) the mass of shaft and the torque of rotor disk are neglig ible.

It is assumed that there is an initial clearance of $\delta$ between the rotor 1 and stator. Compared with one complete period of rotating, the time during rub-impact is so short that the contact between the rotorl and stator can be regarded as elastic impact. Under these assumptions, the rub-impact model is illustrated in Fig. 1(b). When rubbing happens, the radial impacts force $F_{N}$ and the tangential rub force $F_{T}$ can thus be expressed [5] as

$$
\left\{\begin{array}{l}
F_{n}=(r-\delta) k_{r} \\
F_{t}=f F_{n}
\end{array} \quad(\mathrm{r} \geq \delta)\right.
$$

where $k_{r}$ is the radial stiffness of the stator, $\mathrm{f}$ is the friction coefficient between the rotor 1 and stator and $r=\sqrt{x_{1}^{2}+y_{1}^{2}}$ is the radial displacement of the rotor 1 . In xoy reference frame, the forces $F_{x}$ and $F_{y}$ are represented as

$$
\left[\begin{array}{l}
F_{x} \\
F_{y}
\end{array}\right]=-H(r-\delta) \frac{(r-\delta) k_{r}}{r}\left[\begin{array}{cc}
1 & -f \\
f & 1
\end{array}\right]\left[\begin{array}{l}
x_{1} \\
y_{1}
\end{array}\right]
$$

where $\boldsymbol{H}$ is the Heaviside function, can be defined as

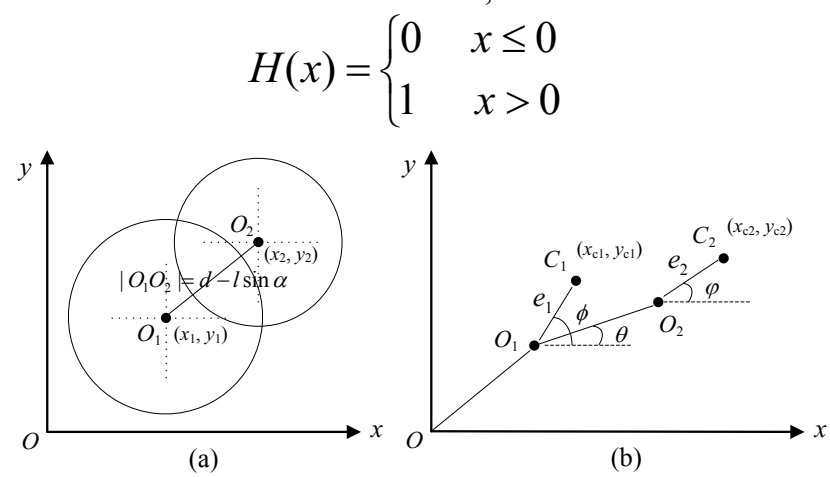

Fig. 2 Coordinate systems of the dual-rotor systems

Figure 2 shows the cross-section of the two rotors and coordinate systems. The relationship of radial displacements between the rotor 1 and rotor 2 can be written as

$$
\left\{\begin{array}{l}
x_{2}=x_{1}+\left|O_{1} O_{2}\right| \cos \theta=x_{1}+(d-l \sin \alpha) \cos \theta \\
y_{2}=y_{1}+\left|O_{1} O_{2}\right| \sin \theta=y_{1}+(d-l \sin \alpha) \sin \theta
\end{array}\right.
$$

where $d$ is the values of parallel misalignment, $\theta$ is the angle of rotor 2 rotating around rotor $1, \theta=\omega t+\theta_{0}$.

The centroid displacements of the rotor 1 and rotor 2 can be respectively calculated as (see Fig. 2b)

$$
\begin{gathered}
\left\{\begin{array}{l}
x_{c 1}=x_{1}+e_{1} \cos \phi \\
y_{c 1}=y_{1}+e_{1} \sin \phi
\end{array}\right. \\
\left\{\begin{array}{l}
x_{c 2}=x_{2}+e_{2} \cos \varphi \cos \alpha \\
y_{c 2}=y_{2}+e_{2} \sin \varphi \cos \alpha
\end{array}\right.
\end{gathered}
$$

where $\left(x_{c 1}, y_{c 1}\right),\left(x_{c 2}, y_{c 2}\right)$ are the centroid coordinates of the rotor 1 and rotor $2, \phi, \varphi$ are the angle of the two rotors rotating respectively, $\phi=\omega t+\phi_{0}, \varphi=\omega t+\varphi_{0}$. 
The total kinetic energy $T$ is constituted of driver kinetic energy $T_{G}$ and the driven kinetic energy $T_{r}$. The system kinetic energy can be written as

$$
T=T_{G}+T_{r}
$$

in which

$$
\begin{aligned}
& T_{G}=\frac{1}{2} m_{1} v_{c 1}^{2}+\frac{1}{2} m_{2} v_{c 2}^{2} \\
& =\frac{1}{2} m_{1}\left(\dot{x}_{1}^{2}+\dot{y}_{1}^{2}+e_{1}^{2} \dot{\phi}^{2}-2 e_{1} \dot{x}_{1} \dot{\phi} \sin \phi\right. \\
& \left.+2 e_{1} \dot{y}_{1} \dot{\phi} \cos \phi\right)+\frac{1}{2} m_{2}\left[\dot{x}_{2}^{2}+\dot{y}_{2}^{2}+\left(e_{2} \cos \alpha\right)^{2} \dot{\varphi}^{2}\right. \\
& \left.-2\left(e_{2} \cos \alpha\right) \dot{x}_{2} \dot{\varphi} \sin \varphi+2\left(e_{2} \cos \alpha\right) \dot{y}_{2} \dot{\varphi} \cos \varphi\right] \\
& T_{r}=\frac{1}{2}\left(J_{1}+m_{1} e_{1}\right) \dot{\phi}^{2}+\frac{1}{2}\left(J_{2}+m_{2} e_{2}\right) \dot{\varphi}^{2}
\end{aligned}
$$

where $J_{1}, J_{2}$ are the inertia moment of the rotor 1 and rotor 2 respec tively.

The elastic potential energy $U$ of the system is defined as

$$
\begin{aligned}
& U=\frac{1}{2} k_{1}\left|O O_{1}\right|^{2}+\frac{1}{2} k_{2}\left|O O_{2}\right|^{2} \\
& =\frac{1}{2} k_{1}\left(x_{1}^{2}+y_{1}^{2}\right)+\frac{1}{2} k_{2}\left(x_{2}^{2}+y_{2}^{2}\right)
\end{aligned}
$$

where $k_{1}, k_{2}$ are the bearing stiffness of the rotor 1 and rotor 2 respectively.

It is assumed that only the damping $c$ and the external exciting forces of the rotor 1 are not considered. So the generalized force for the dual-rotor system can be written as

$$
\left\{\begin{array}{l}
Q_{x}=-c \dot{x}_{1}+F_{x} \\
Q_{y}=-c \dot{y}_{1}+F_{y}
\end{array}\right.
$$

Upon substituting the kinetic and potential energy expressions into the Lagrange equation, performing partial differentiation and manipulation, if $x=x_{1}, y=y_{1}$, the system equation of motion is found by Equation (1) $\sim$ Equation (10) as

$$
\left\{\begin{array}{l}
\left(m_{1}+m_{2}\right) \ddot{x}+c \dot{x}+\left(k_{1}+k_{2}\right) x=m_{1} e_{1} \omega^{2} \cos \phi+ \\
m_{2} e_{2} \omega^{2} \cos \alpha \cos \varphi+m_{2}(d-l \sin \alpha) \omega^{2} \cos \theta \\
-k_{2}(d-l \sin \alpha) \cos \theta+F_{x} \\
\left(m_{1}+m_{2}\right) \ddot{y}+c \dot{y}+\left(k_{1}+k_{2}\right) y=m_{1} e_{1} \omega^{2} \sin \phi+ \\
m_{2} e_{2} \omega^{2} \cos \alpha \sin \varphi+m_{2}(d-l \sin \alpha) \omega^{2} \sin \theta \\
-k_{2}(d-l \sin \alpha) \sin \theta+F_{y}
\end{array}\right.
$$

In right side of the equation(11), the preceding two items are respectively centrifugal inertial force of the rotor 1 and rotor 2 as the shaft is rotating, and the centrifugal inertial force of the rotor 2 caused by parallel and angular misalignment is given in the third item, the fourth is the force exerted by the bearing and the last item is the rub-impact force mentioned above. It can be concluded clearly that the mass eccentricity, parallel misalignment and stiffness are the main factors on the stability of the rotor system.

For the convenience of calculating composition and results analysis, the dimensionless transformations are given as follows:

$$
X=\frac{x}{\delta}, \quad Y=\frac{y}{\delta}, \quad D=\frac{d}{\delta}
$$

\section{Simulation result and analysis}

Due to the strongly nonlinear characteristics of the rubimpacting rotor system, the vibration responses are quite complicated. Numerical analysis is carried out by the fourth-order Runge-Kutta method. During the calculation a smaller integration step has to be chosen to ensure that a stable solution will be obtained as well as to avoid the numerical divergence at the point where derivatives of the rub-impact forces are discontinuous.

The values for the computational parameters are as follows:

$m_{1}=3.5 \times 10^{5} \mathrm{~kg}, \quad m_{2}=3.0 \times 10^{5} \mathrm{~kg}, \quad c=2.1 \times 10^{6} \mathrm{~N} \cdot \mathrm{s} / \mathrm{m}$, $k_{1}=1.0 \times 10^{7} \mathrm{~N} / \mathrm{m}, \quad k_{2}=1.2 \times 10^{7} \mathrm{~N} / \mathrm{m}, \quad k_{r}=2.5 \times 10^{8} \mathrm{~N} / \mathrm{m}$, $\delta=2.0 \mathrm{~mm}, f=0.01, \phi_{0}=0.5 \mathrm{rad}, \varphi_{0}=0.3 \mathrm{rad}, \theta_{0}=0.1 \mathrm{rad}$, $\omega=35 \mathrm{rad} / \mathrm{s}$.

\subsection{Influence of parallel misalignment}
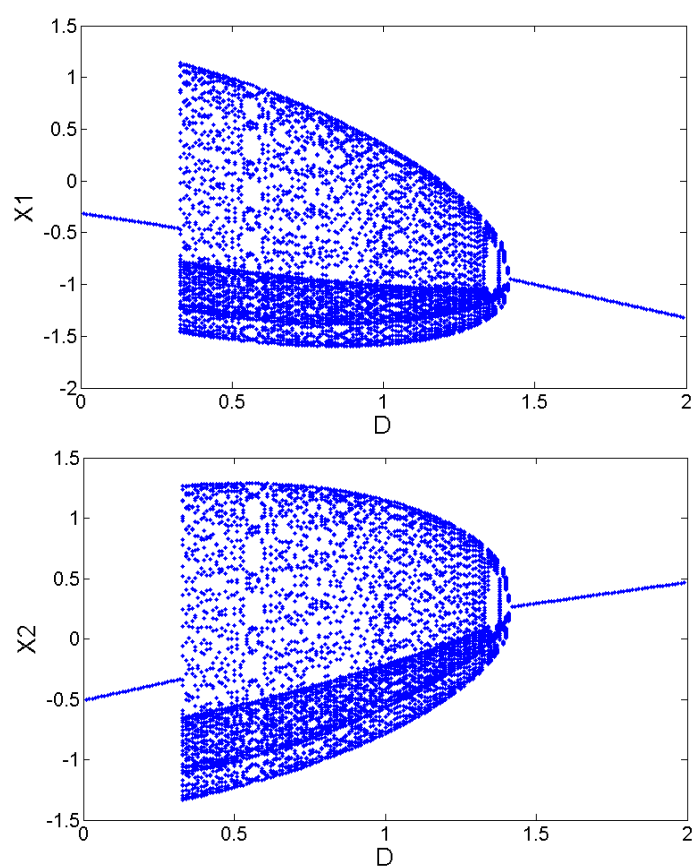

Fig. 3. Bifurcation diagrams of the rotor 1 and rotor 2 with the parallel misalignment $\mathrm{D}$ as a control parameter 
Figure 3 shows the bifurcation diagrams of the rotor 1 and rotor 2 using the parallel misalignment $D$ as the control parameter with mass eccentricity $e_{1}=0.8$ $\mathrm{mm}, e_{2}=1.0 \mathrm{~mm}$ and $\alpha=0.0001 \mathrm{rad}$. It can be seen that the rotor responses contain periodic and quasiperiodic motions at $D=0 \sim 2$. To illustrate the various process of motion, Fig. 4 shows the nonlinear characteristics of the dual-rotor system with the plots of Poincaré maps, axis orbits, time histories and amplitude spectrum diagrams at different $D$. When parallel misalignment is small, the rub phenomenon does not occur, the motion is synchronous with period-one, and only one point is correspondingly displayed in the bifurcation diagram. It can also be clearly seen form the spectrum that a single spectrum line can be observed at the $1 \mathrm{X}$ component and zero at other positions. Fig. 4(a) shows the dynamic characteristics at $D=0.2$. With the increase of the parallel misalignment, rub does occur between the rotor 1 and the stator. When $D>0.32$, the rub-impact happens and the rotor motion becomes quasi-periodic. At $D=0.57$, there are some isolated points in the Poincaré map and periodic motions in the axis orbit. The beat vibration is observed on this time history and the amplitude of the $1 / 3 \mathrm{X}$ component is bigger to that of $1 \mathrm{X}$ component as shown in Fig. 4(b). The more greatly the parallel misalignment increases, some changes can be seen when $D=1.36$ as shown in Fig. 4(c). In this case there is a closed curve in the Poincare map and the vibration waveforms in some periods are not so regular which indicates that the rubimpact has become more serious. As $D$ exceeds 1.41, the motion comes into period-one again because the dual-rotor system reaches dynamic balance. Although the two rotors are rigidly coupled by the shaft, dynamic characteristics of the rotor 1 with the rubimpact force are more complicated than those of the rotor 2 such as axis orbits and time histories.
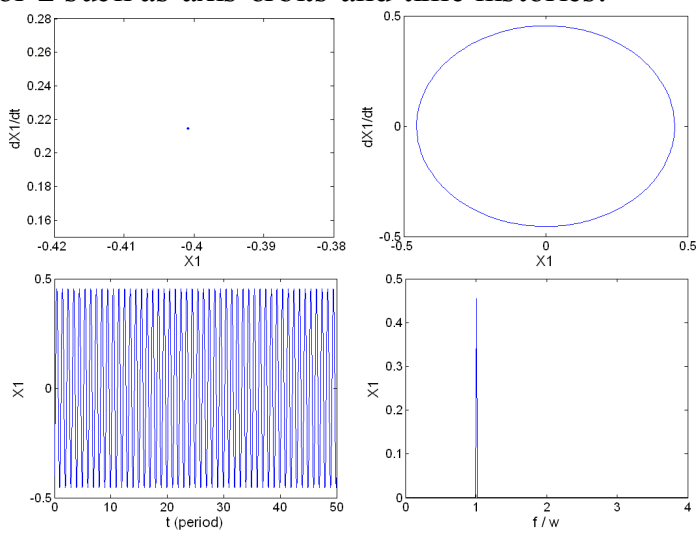

(a) $D=0.2$
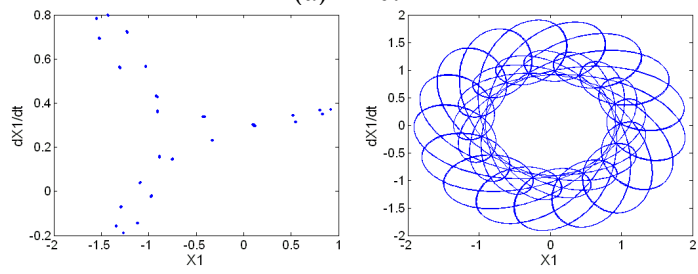
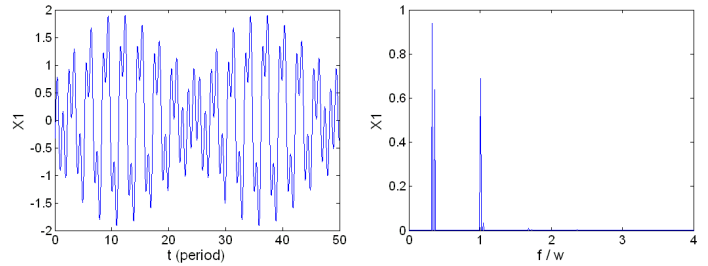

(b) $D=0.57$
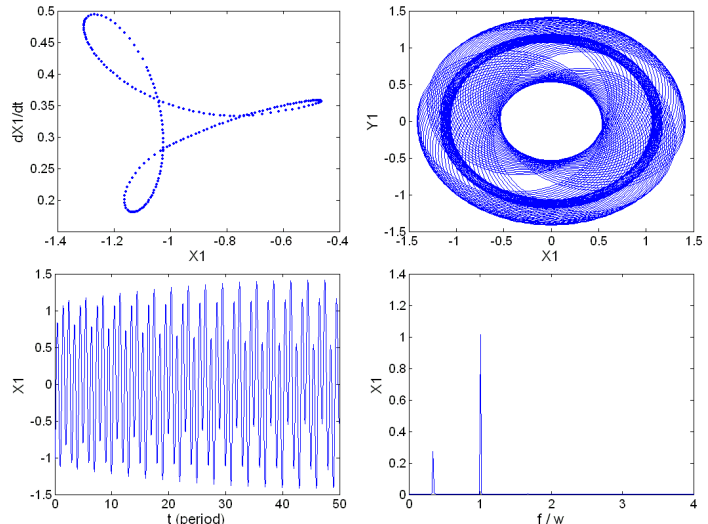

(c) $D=1.36$

Fig. 4. Poincaré maps, axis orbits, time-histories and amplitude spectrum diagrams on the response of the rotor 1 for different parallel misalignment

From amplitude spectrums of Fig. 4(a)-(d), we find some low frequencies with large amplitude besides $1 \mathrm{X}$ component, especially at $1 / 3 \mathrm{X}$ component. Amplitude of the rotor 1 at $1 \mathrm{X}$ component is increasing constantly. At the same time, amplitudes of low frequencies of the two rotors are decreasing. Responses of sub-harmonic for the dual-rotor system caused by the coupling faults with misalignment and rub-impact make amplitudes of low frequencies bigger than that at $1 \mathrm{X}$ component in some conditions. The phenomenon can be defined as a typical feature for the coupling faults. In addition, it also could provide important theory basis to diagnose the kind of fault.

\subsection{Influence of angular misalignment}

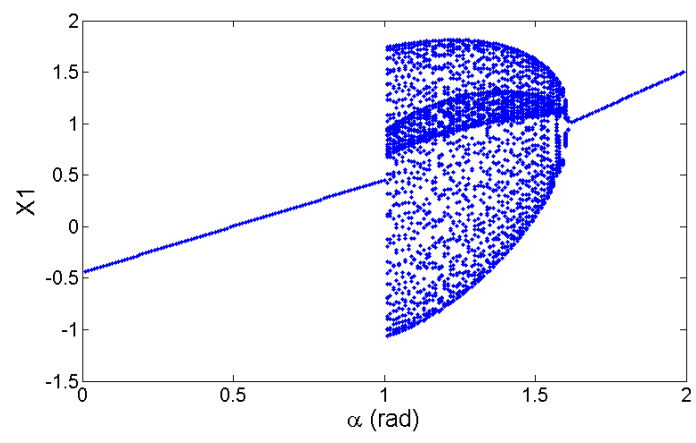




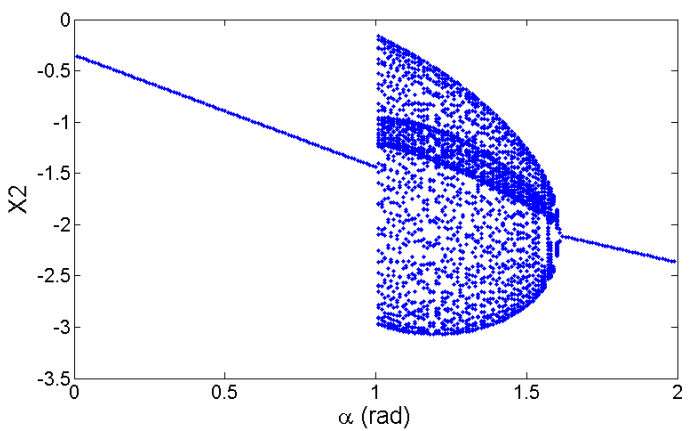

Fig. 5. Bifurcation diagrams of the rotor 1 and rotor 2 response with the angular misalignment $\mathrm{D}$ as a control parameter

Fig. 5 shows the bifurcation diagrams and its magnification of angular misalignment $\alpha$ on the response of the rotor 1 and rotor 2 with mass eccentricity $e_{1}=0.8 \mathrm{~mm}, e_{2}=1.0 \mathrm{~mm}$ and $D=0.1$. It can be seen that system responses exhibit periodic, there periodic and quasi-periodic motions at the interval of $(0<\alpha<0.002) \mathrm{rad}$. At $\alpha<1.0 \times 10^{-3} \mathrm{rad}$, the system response displays steady-sate synchronous motion with period-one as shown in Fig. 6(a), the vibration amplitude of the dual-rotor system is small and rub phenomenon generally does not occur. As the imbalance increases, the vibration amplitude of the dual-rotor system becomes bigger and the rub phenomenon occurs between the rotor 1 and stator. At $\alpha>1.0 \times 10^{-3} \mathrm{rad}$, the system response comes into quasi-periodic motion and exhibits a wider range of quasi-periodic motion as the control parameters vary. Fig. 6(b) illustrate the dynamic characteristics at $\alpha=1.4 \times 10^{-3} \mathrm{rad}$. It can be seen from that the closed from of the attractor is decomposed in the Poincare map and two discrete frequency components arise in the amplitude spectrum. All of these give proof of that the motion is quasi-periodic. The more greatly the angular misalignment increases, the motion becomes a sub-synchronous vibration with period-three and its attractor is three isolated points, exhibiting three different circular rings in the axis orbit and amplitudes in time history correspondingly as shown in Fig. 6(c). $1 / 3 \mathrm{X}$ component can be clearly seen from the spectrum except the $1 \mathrm{X}$ component. At $\alpha>1.61 \times 10^{-3}$ $\mathrm{rad}$, the motion comes into period-one again.
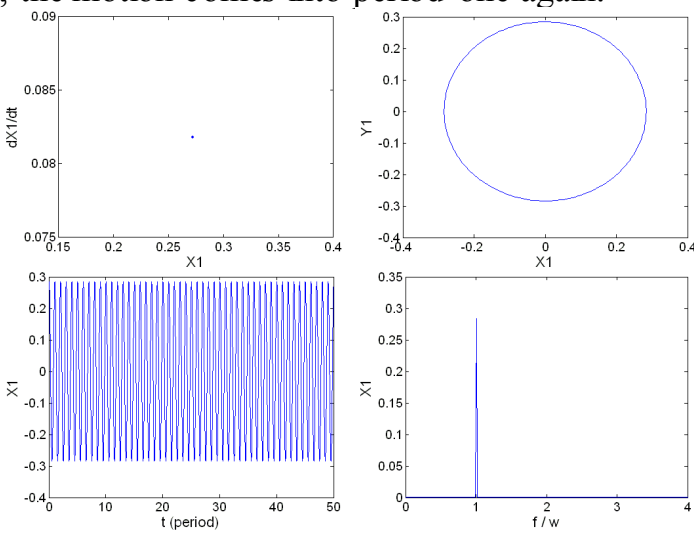

(a) $\alpha=0.8 \times 10^{-3} \mathrm{rad}$
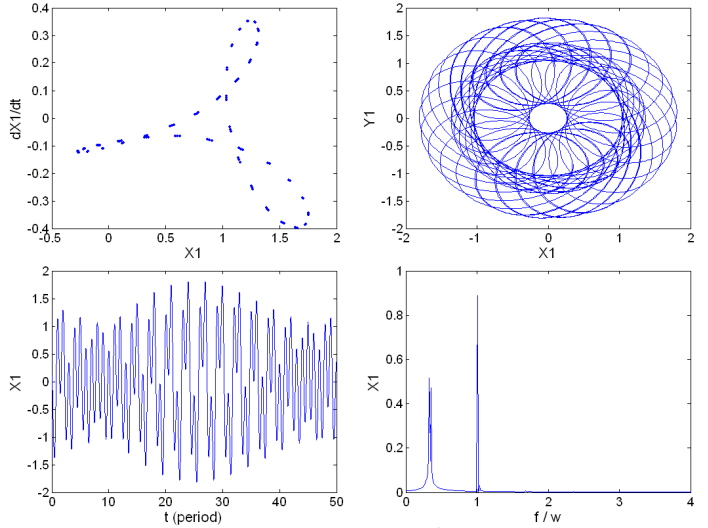

(b) $\alpha=1.4 \times 10^{-3} \mathrm{rad}$
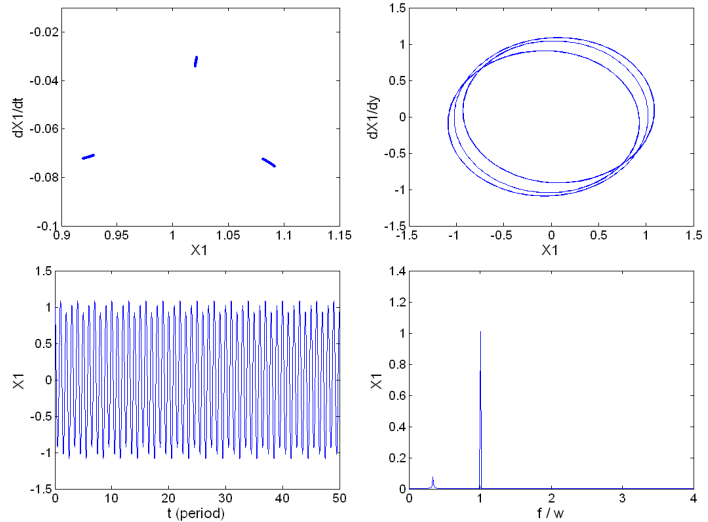

(c) $\alpha=1.61 \times 10^{-3} \mathrm{rad}$

Fig. 6. Poincaré maps, axis orbits, time-histories and amplitude spectrum diagrams on the response of the rotor 1 for different angu lar misalignment

Comparis on between Figure 3 and Figure 5 reveals that dynamic characteristics of the dual-rotor system are very similar as the increase of parallel misalign ment and angular misalignment. However, the changes of angular misalignment can effect the centrifugal inertial of the rotor 1 , so there are some distinctive responses of the dual-rotor system such as axis orbits and amplitude spectrum. It can be also observed that the influence of parallel misalignment is stronger than that of angular misalignment.

\section{Conclusions}

In this study, according to fault problems of the dualrotor system local rubbing caused by mass eccentricity, a model of the dual-rotor system with coupling faults of misalignment and rub-impact has been developed. Some non-linear analysis methods were employed to explore dynamic behaviors of the discussed system, including Poincaré maps, axis orbits, time histories, amplitude spectrum and bifurcation diagrams. The influence of parameters such as parallel misalignment, angular misalignment and mass eccentricity on the dynamic characteristics of the dual-rotor system is studied theoretically in detail. It can be observed that the system exhibits various forms of periodic, three-periodic and quasi-periodic. The simulation results show that there are some low-frequency components with large amplitude in the amplitude spectrum, especially at the $1 / 3 \mathrm{X}$ component and beat vibration phenomenon occurs in the 
time history for quasi-periodic. Although the rotor 1 and rotor 2 are rigid coupled, dynamic characteristics of two rotors are some distinctive. It is found the mass eccentricity can obviously change radial vibration of the dual-rotor system. With the increase of mass eccentricity, system responses mainly alternate between periodic and quasi-periodic motions. It will be seen from th is that the performances of the dual-rotor system are significantly influenced by the characteristics of the misalignment. It is imperative to understand the nonlinear behavior of the dual-rotor system with the coupling faults. The purpose of the further research is effectively to diagnose the coupling faults, reduce the failure and optimize the design of the dual-rotor r system.

\section{Acknowledgments}

This work has been supported by Project of Program of National Natural Science Foundation of China (No.50539140).

\section{References}

1. Lees AW. Misalignment in rigidly coupled rotors. Journal of Sound and vibration, 2007, 305: 261-271.

2. Li M, Yu L. Analysis of the coupled lateral torsional vibration of a rotor-bearing system with a misalignment gear coupling. Journal of Sound and vibration, 2001, 243(2): 283-300.

3. Hamdi T, Selcuk E, Ibrahim U. Experimental analysis on fault detection for coupled rotor-bearing system. Measurement, 2013, 46(1): 336-344.

4. Li QK, Liao MF, Jiang YF. The vibration features analysis of twin spool rotor with misalign ment fault. Mechanical Science and Technology for Aerospace Engineering, 2015, 33(12): 1916-1920.

5. Li ZG, Li M. Non-linear dynamics of a flexible multi-rotor bearing system with a fault of parallel misalignment. Applied Mechanics and Materials, 2012, 138-139: 104-110.

6. Wan C, Jian C, Chen CK. Chaos of rub-impact rotor supported by bearings with nonlinear suspension. Tribology International, 2009, 42: 426-439.

7. Al-Hussain KM, Redmond I. Dynamic response of two rotors connected by rigid mechanical coupling with parallel misalignment. Journal of Sound and vibration, 2002, 249(3): 483-498.

8. Xia SB, Zhang XJ, Liu ZS, et al. A survey of research on misalignment of rotary machinery. Journal of vibration, Measurement and Diagnosis, 1998, 18(3): 157-160.

9. $\mathrm{Hu} \mathrm{W}$, Miah $\mathrm{H}$, Feng NS, et al. A rig for testing lateral misalignment effects in a flexible rotor supported on three or more hydrodynamic journal bearings. Tribology International, 2000, 33: 197-204.

10. Han J, Shi LD. Study on kinematic mechanis $m$ of misalignment fault of rotor system connected by gear coupling. Journal of Vibration Engineering, 2004, 17(4): 416-420.

11. Wang YB. Shaft misalignment induced vibration in high capacity steam-turbine generator units. Power Engineering, 2004, 24(6): 768-774.

12. Al-Hussain KM. Dynamic stability of two rigid rotors connected by a flexible coupling with angular misalignment. Journal of Sound and Vibration, 2003, 266: 217-234.

13. Bouaziz S, Hili MA, Mataar M, et al. Dynamic behaviour of hydrodynamic journal bearings in presence of rotor spatial angular misalignment. Mechanism and Machine Theory, 2009, 44: 15481559.

14. Saavedra PN, Ramirez DE. Vibration analysis of rotors for the identification of shaft misalignment Part II : Experimental validation. Journal of Mechanical Engineering Science, 2004, 218(c): 987999.

15. Patel TH, Darpe AK. Experimental investigations on vibration response of misalignment rotors. Mechanical System and Signal Processing, 2009, 23: 2236-2252.

16. Liu Y, Tai XY, Yao HL, et al. Study on misalignment-rubbing coupling fault of dual-disk rotor-bearing system including the impact of motor coupling. Journal of Vibration, Measurement and Diagnos is, 2013; 33(5): 819-823.

17. Zhu YY, Zhou QZ, Wang DS. Vibration characteristics of a rub-impact rotor system with an angular misalignment under the unsteady oil-film forces. Journal of Wuhan University (Natural Science Edition), 2005, 283: 621-643.

18. Shen XY, Jia JH, Zhao M. Effect of parameters on the rubbing condition of an unbalanced rotor system with initial permanent deflection. Archive of Applied Mechanics, 2007, 77(12): 883-892. 